\title{
Serum Sodium Levels Predict Mortality in Elderly Acute Kidney Injury Patients: A Retrospective Observational Study
}

This article was published in the following Dove Press journal: International Journal of General Medicine

\author{
Qinglin $\mathrm{Li}^{\mathrm{I}} *$ \\ Yan Wang ${ }^{2} *$ \\ Zhi Mao' \\ Hongjun Kang' \\ Feihu Zhou (D) ${ }^{1,3}$ \\ 'Department of Critical Care Medicine, \\ The First Medical Centre, Chinese PLA \\ General Hospital, Beijing, I00853, \\ People's Republic of China; ${ }^{2}$ Department \\ of Health Care, The Second Medical \\ Centre, Chinese PLA General Hospital, \\ Beijing, 100853, People's Republic of \\ China; ${ }^{3}$ Chinese PLA General Hospital \\ National Clinical Research Center for \\ Geriatric Diseases, Beijing, 100853, \\ People's Republic of China
}

*These authors contributed equally to this work
Correspondence: Feihu Zhou

Department of Critical Care Medicine,

The First Medical Centre, Chinese PLA

General Hospital, Beijing, 100853,

People's Republic of China

Tel + 86-|0-66938|48

Fax + 86-10-88219862

Email feihuzhou30I@I26.com
Purpose: We examined the relationship between different levels of serum sodium and mortality among elderly patients with acute kidney injury (AKI).

Methods: We retrospectively enrolled elderly patients from Chinese PLA General Hospital from 2007, to 2018. All-cause mortality was examined according to eight predefined sodium levels: $<130.0 \mathrm{mmol} / \mathrm{L}, 130.0-134.9 \mathrm{mmol} / \mathrm{L}, 135.0-137.9 \mathrm{mmol} / \mathrm{L}, 138.0-141.9 \mathrm{mmol} / \mathrm{L}$, $142.0-144.9 \mathrm{mmol} / \mathrm{L}, 145.0-147.9 \mathrm{mmol} / \mathrm{L}, 148.0-151.9 \mathrm{mmol} / \mathrm{L}$, and $\geq 152.0 \mathrm{mmol} / \mathrm{L}$. We estimated the risk of all-cause mortality using a multivariable adjusted Cox analysis, with a normal sodium level of $135.0-137.9 \mathrm{mmol} / \mathrm{L}$ as a reference.

Results: In total, 744 patients were suitable for the final evaluation. After 90 days, the mortality rates in the eight strata were $36.1,27.8,19.6,24.4,30.7,48.6,52.8$, and $57.7 \%$, respectively. In the multivariable adjusted analysis, patients with sodium levels $<130.0$ mmol/L (HR: 2.247; 95\% CI: 1.117-4.521), from 142.0 to $144.9 \mathrm{mmol} / \mathrm{L}$ (HR: 1.964 ; 95\% CI: $1.100-3.508$ ), from 145.0 to $147.9 \mathrm{mmol} / \mathrm{L}$ (HR: $2.942 ; 95 \% \mathrm{CI}: 1.693-5.114$ ), from 148.0 to $151.9 \mathrm{mmol} / \mathrm{L}$ (HR: 3.455 ; $95 \% \mathrm{CI}: 2.009-5.944$ ), and $\geq 152.0 \mathrm{mmol} / \mathrm{L}$ (HR: 3.587; 95\% CI: 2.151-5.983) had an increased risk of all-cause mortality. After 1 year, the mortality rates in the eight strata were $58.3,47.8,33.7,38.9,45.5,64.3,69.4$, and $78.4 \%$, respectively. Patients with sodium levels $<130.0 \mathrm{mmol} / \mathrm{L}$ (HR: $1.944 ; 95 \%$ CI: $1.125-3.360$ ), from 142.0 to $144.9 \mathrm{mmol} / \mathrm{L}$ (HR: 1.681 ; $95 \% \mathrm{CI}$ : $1.062-2.660$ ), from 145.0 to $147.9 \mathrm{mmol} /$ L (HR: $2.631 ; 95 \%$ CI: $1.683-4.112$ ), from 148.0 to $151.9 \mathrm{mmol} / \mathrm{L}$ (HR: $2.411 ; 95 \% \mathrm{CI}$ : $1.552-3.744$ ), and $\geq 152.0 \mathrm{mmol} / \mathrm{L}$ (HR: 3.037; 95\% CI: $2.021-4.563$ ) had an increased risk of all-cause mortality.

Conclusion: Sodium levels outside the interval of $130.0-141.9 \mathrm{mmol} / \mathrm{L}$ were associated with increased risks of 90-day mortality and 1-year mortality in elderly AKI patients.

Keywords: serum sodium, acute kidney injury, elderly, mortality

\section{Introduction}

Acute kidney injury (AKI) is a common clinical acute critical illness that is frequently encountered in the elderly population. ${ }^{1-3}$ The disorder is generally characterized by an abrupt deterioration in renal function (RF) that disrupts metabolic, electrolyte and fluid homeostasis over a period of hours to days. ${ }^{4-6}$ Aging kidneys undergoing structural and functional changes that decrease autoregulatory capacity, systemic vasculature, and the immunological system render the elderly population highly susceptible to AKI. ${ }^{7,8}$ Previous studies of AKI in the elderly population mostly examined all-cause mortality, renal prognosis, or cardiovascular 
events. ${ }^{9,10}$ However, little work has been done on investigating electrolyte imbalances in elderly AKI patients, such as dysnatremia and its association with mortality. ${ }^{11}$

The kidneys play a central role in sodium homeostasis, and their functional decline leads to electrolyte disorders. ${ }^{12}$ Recently, Gao reported that compared with the reference group (136.0-144.9 mmol/L), AKI patients at the time of hospital admission with hyponatremia $(<136.0 \mathrm{mmol} / \mathrm{L})$ or hypernatremia ( $\geq 145.0 \mathrm{mmol} / \mathrm{L}$ ) had higher 90 -day mortality rates. ${ }^{12}$ However, the independent or synergistic prognostic effects of abnormal serum sodium levels remain less well studied among elderly AKI patients. In addition, the normal ranges of serum sodium levels that are applicable to such patients are still unknown.

Therefore, identifying the clinically significant normal ranges of serum sodium is an important issue for clinicians when making decisions regarding elderly AKI patients with dysnatremia.

\section{Patients and Methods}

This was a retrospective observational study conducted at the Chinese PLA General Hospital National Clinical Research Center for Geriatric Diseases (Beijing, China). All patients aged $\geq 75$ years with normal renal function who were admitted to general ward between January 2007 and December 2018 were enrolled. The study design was approved by the Clinical Ethics Committee of the Chinese PLA General Hospital (number: S2017-054-01). The requirement to obtain written informed consent from each patient was waived because this was an observational retrospective study. The patients' information were anonymous and non identifiable. This study was conducted in accordance with the declaration of Helsinki. All admissions were screened and evaluated for AKI and categorized according to the Kidney Disease: Improving Global Outcomes (KDIGO) criteria.

Demographic and basic data were obtained from the medical records. Baseline serum sodium levels, and the sodium level at the time of AKI diagnosis were recorded. Other laboratory data of interest included baseline serum creatinine (Scr) level, the Scr level at the time of AKI diagnosis, and the levels of blood urea nitrogen (BUN), uric acid, blood glucose (BG), electrolytes ( $\mathrm{K}, \mathrm{Ca}, \mathrm{P}$, and $\mathrm{Mg}$ ), C-reactive protein (CRP), albumin, prealbumin, and hemoglobin.

The exclusion criteria were as follows: patients who had been previously diagnosed with chronic kidney disease (CKD), a hospital stay $<48 \mathrm{~h}$, patients with no Scr data or only one Scr test, patients with insufficient medical records, and patients who died within $48 \mathrm{~h}$ of admission.

\section{Definitions}

The 2012 KDIGO-defined Scr criteria were used to identify and classify AKI. ${ }^{13}$ The CKD Epidemiology Collaboration method was used to calculate the baseline estimated glomerular filtration rate (eGFR) ${ }^{14}$ The baseline Scr level was the most recent measure taken in the 1-3 months before admission for AKI. ${ }^{15}$ Sepsis was defined according to the Surviving Sepsis Campaign Bundle: 2018 update. $^{16}$

The normal sodium reference range $(135.0-145.0 \mathrm{mmol} /$ L) at the time of AKI diagnosis was divided into three intervals. Furthermore, five intervals outside the normal sodium reference range were defined: one interval contained patients with a serum sodium level below $130.0 \mathrm{mmol} / \mathrm{L}$, one contained those with a serum sodium level from $130.0 \mathrm{mmol} / \mathrm{L}$ to $134.9 \mathrm{mmol} / \mathrm{L}$, one contained those with a serum sodium level from $145.0 \mathrm{mmol} / \mathrm{L}$ to $147.9 \mathrm{mmol} / \mathrm{L}$, one contained those with a serum sodium level from $148.0 \mathrm{mmol} / \mathrm{L}$ to $151.9 \mathrm{mmol} /$ $L$ and last, one interval contained patients with a serum sodium above $152.0 \mathrm{mmol} / \mathrm{L}$. Thus, this study contains eight sodium intervals, where the reference interval was defined as a sodium level from 135.0 to $137.9 \mathrm{mmol} / \mathrm{L}$ based on the analysis, which confirmed that the lowest mortality risk was found in this range (Table 1).

\section{Outcomes}

The outcome of the study was all-cause mortality within 90 days and within 1 year after AKI diagnosis.

\section{Statistical Analysis}

Continuous variables are presented as the mean \pm standard deviation for parametric variables or as the median with interquartile range (with 25 th and 75 th percentiles) for nonparametric variables. Categorical variables are presented as numbers (n) or percentages (\%). Group comparisons were conducted using ANOVA or the KruskalWallis $H$-test for continuous variables and Pearson's chisquare or Fisher's exact test for categorical variables. Prognostic survival factors were identified using the Cox proportional hazards regression model. Survival probability was estimated using the Kaplan-Meier method for the eight sodium intervals, and curves were compared among groups using the Log rank test. A $P$-value $<0.05$ was considered significant. Statistical analyses were performed using SPSS version 21.0 for Windows software (SPSS Inc., Chicago, IL, USA). 


\begin{tabular}{|c|c|c|c|c|c|c|c|c|c|c|c|}
\hline$\frac{0}{\frac{0}{3 ⿻}}$ & : & $\stackrel{\text { o̊g }}{\circ}$ & 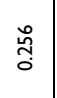 & 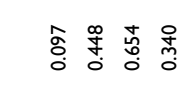 & $\begin{array}{l}\overline{8} \\
\dot{0} \\
\mathrm{v}\end{array}$ & $\begin{array}{l}\bar{o} \\
\dot{Q} \\
\mathrm{v}\end{array}$ & : & 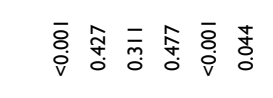 & 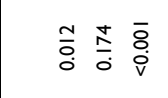 & 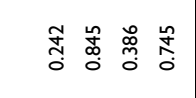 & 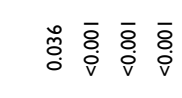 \\
\hline 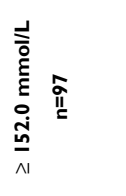 & 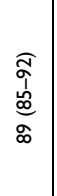 & 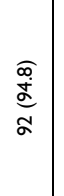 & $\begin{array}{l}\text { जे } \\
\text { ثें } \\
\text { in }\end{array}$ & 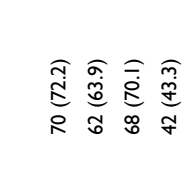 & 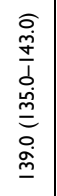 & $\left|\begin{array}{l}0 \\
0 \\
0 \\
0 \\
0 \\
0 \\
0 \\
0 \\
0 \\
0 \\
0\end{array}\right|$ & 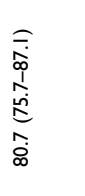 & 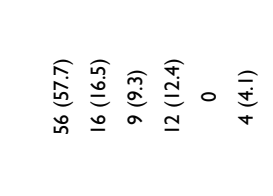 & 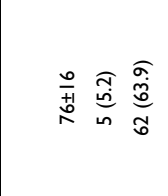 & 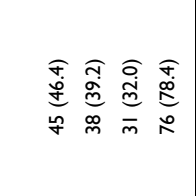 & 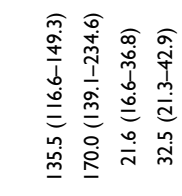 \\
\hline 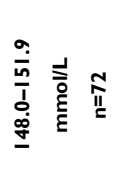 & 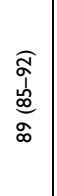 & 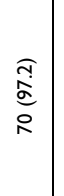 & $\begin{array}{l}\text { i } \\
\text { 苦 } \\
\text { in }\end{array}$ & 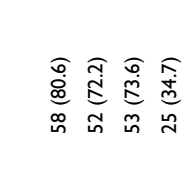 & 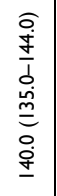 & 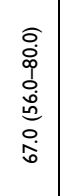 & 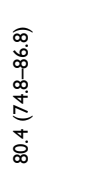 & 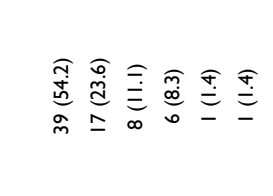 & 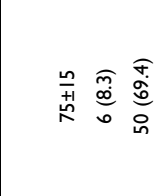 & 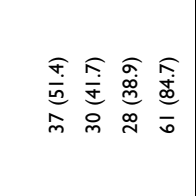 & 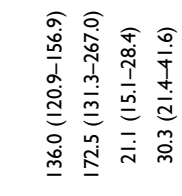 \\
\hline 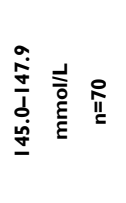 & $\begin{array}{l}\widehat{\widehat{\sigma}} \\
\hat{0} \\
\text { o. } \\
\stackrel{0}{\alpha}\end{array}$ & 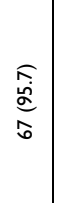 & 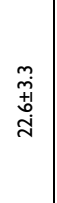 & 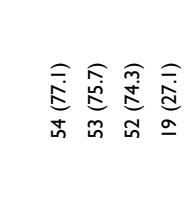 & 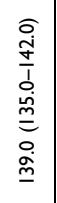 & 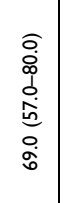 & 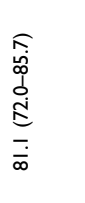 & 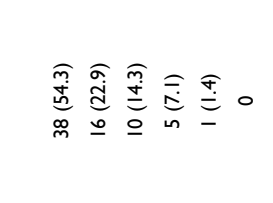 & 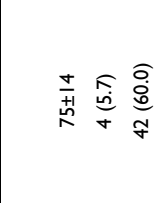 & 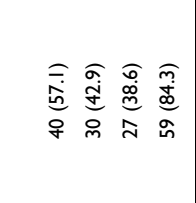 & 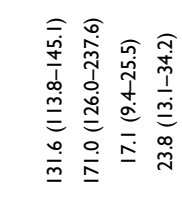 \\
\hline 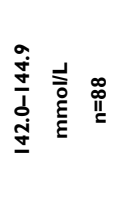 & 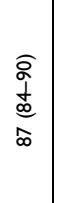 & 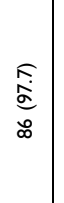 & 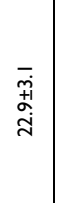 & 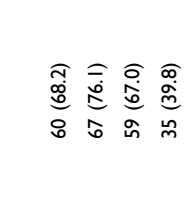 & 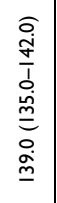 & 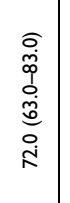 & 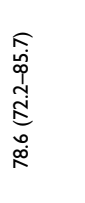 & 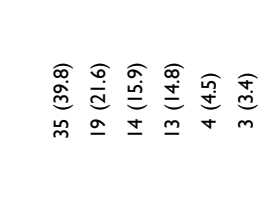 & 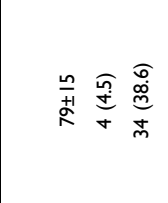 & 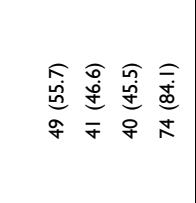 & 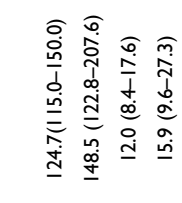 \\
\hline 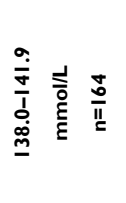 & $\begin{array}{l}\bar{a} \\
\hat{\alpha} \\
\infty \\
\infty \\
\infty \\
\infty\end{array}$ & 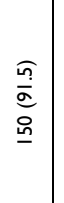 & $\begin{array}{c}\stackrel{+}{\sim} \\
\stackrel{\sim}{+} \\
\underset{\sim}{m}\end{array}$ & 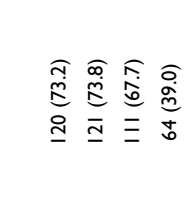 & 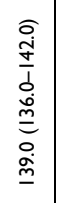 & 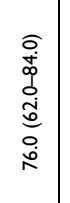 & 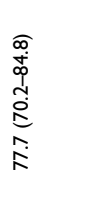 & 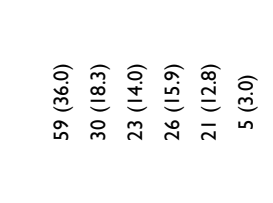 & 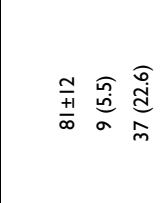 & 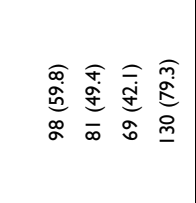 & 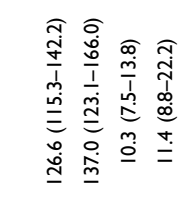 \\
\hline 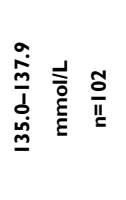 & $\begin{array}{l}\widehat{\overline{\hat{a}}} \\
\stackrel{\infty}{\infty} \\
\hat{\infty}\end{array}$ & 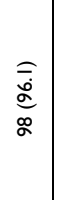 & 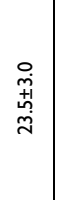 & 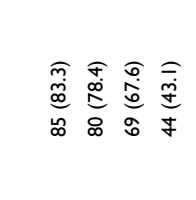 & 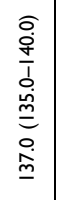 & 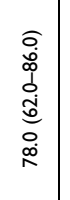 & 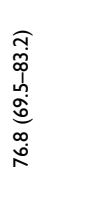 & 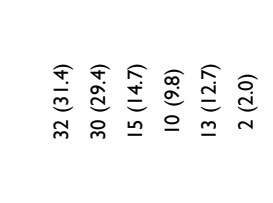 & 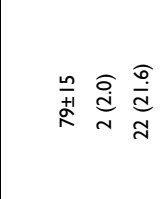 & 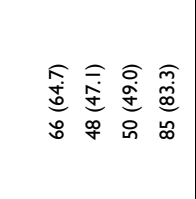 & 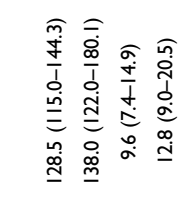 \\
\hline 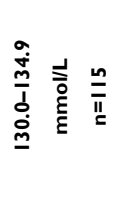 & $\begin{array}{l}\overline{\overline{0}} \\
\overline{0} \\
0 \\
\hat{\infty}\end{array}$ & 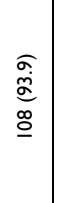 & 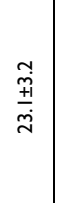 & 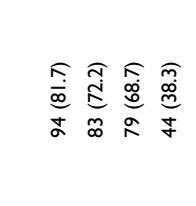 & 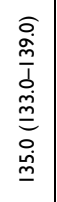 & 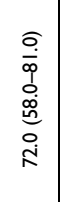 & 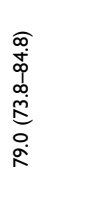 & 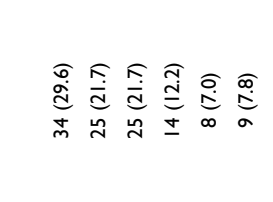 & 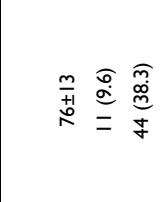 & 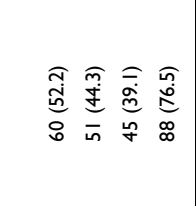 & 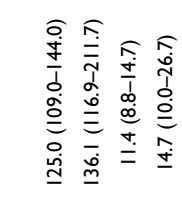 \\
\hline 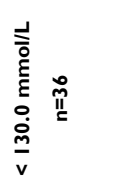 & 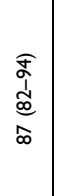 & 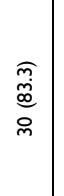 & $\begin{array}{c}\hat{i} \\
\dot{N} \\
\underset{\sim}{n}\end{array}$ & 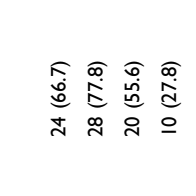 & 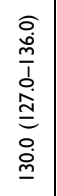 & 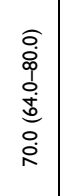 & 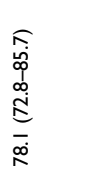 & 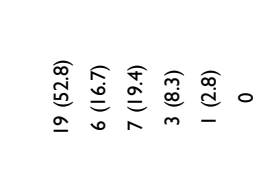 & 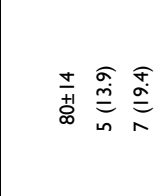 & 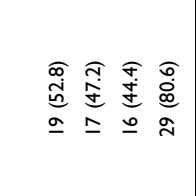 & 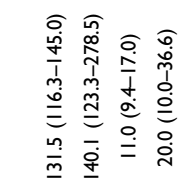 \\
\hline$\frac{\sqrt{4}}{\frac{y}{4}}$ & $\begin{array}{l}\widehat{\overline{\bar{a}}} \\
\dot{\Phi} \\
\infty \\
\infty \\
\infty\end{array}$ & $\begin{array}{l}\widehat{\widehat{z}} \\
\bar{\Phi} \\
\overline{2}\end{array}$ & $\begin{array}{l}\bar{p} \\
\text { 尊 } \\
\text { i }\end{array}$ & 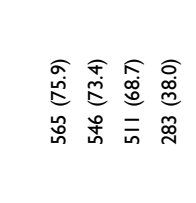 & 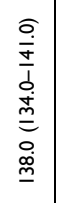 & 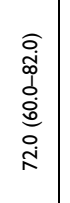 & 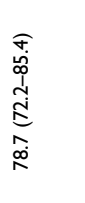 & 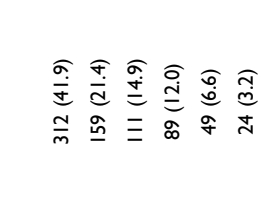 & 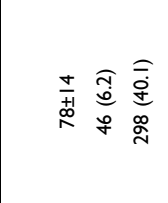 & 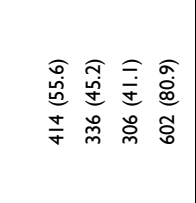 & 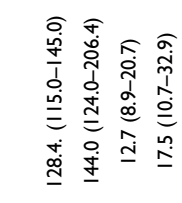 \\
\hline 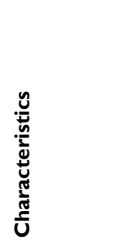 & 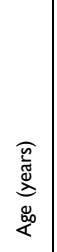 & $\begin{array}{l}\frac{x}{0} \\
\frac{d}{\tilde{c}} \\
\frac{\tilde{c}}{\Sigma}\end{array}$ & 定 & 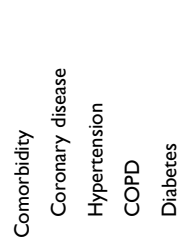 & 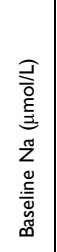 & 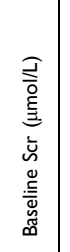 & 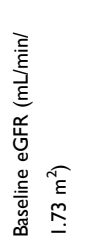 & 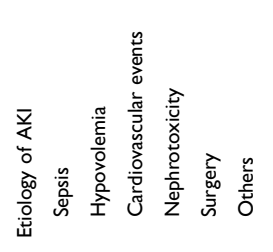 & 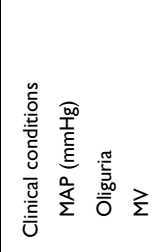 & 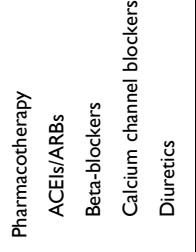 & 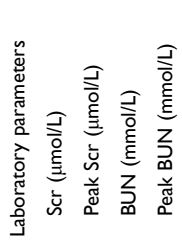 \\
\hline
\end{tabular}




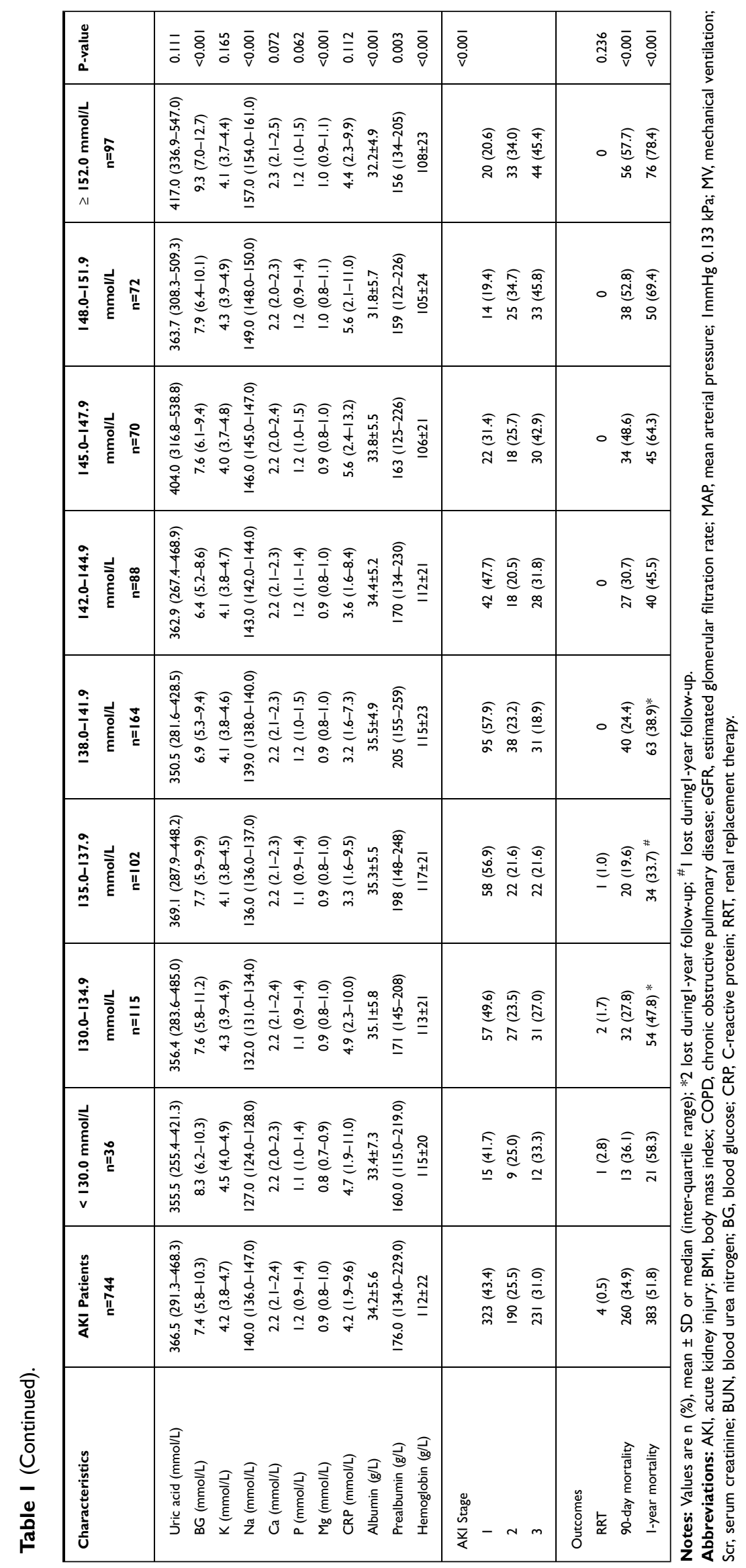




\section{Results}

\section{Study Population}

In the period 2007-2018, a total of 3861 elderly patients (aged $\geq 75$ years) were hospitalized at the National Clinical Research Center for Geriatric Diseases, 760 of whom developed AKI during hospitalization. Of these patients, 13 were excluded because of a length of hospital stay of less than 48 hours, 3 were excluded because of missing data that was required for this study, and 744 AKI patients were suitable for the final analysis. The median age of the 744 participants was 88 years, and the majority (701, 94.2\%) were male. Among all patients, 260 (34.9\%) died within 90 days. During the 1-year follow-up, 5 patients were lost, and 383 (51.8\%) died.

\section{General Conditions and Clinical Characteristics According to Sodium Levels}

A summary of the baseline characteristics of the study population is provided in Table 1 . The overall median serum sodium level when AKI was diagnosed was 140.0 $\mathrm{mmol} / \mathrm{L}(136.0-147.0 \mathrm{mmol} / \mathrm{L})$. Thirty-six patients were in the low sodium group $(<130.0 \mathrm{mmol} / \mathrm{L}), 115$ in the sodium group $130.0-134.9 \mathrm{mmol} / \mathrm{L}, 102$ in the sodium group 135.0-137.9 mmol/L, 164 in the sodium group 138.0-141.9 $\mathrm{mmol} / \mathrm{L}, 88$ in the sodium group $142.0-144.9 \mathrm{mmol} / \mathrm{L}, 70$ in the sodium group 145.0-147.9 mmol/L, 72 in the sodium group 148.0-151.9 $\mathrm{mmol} / \mathrm{L}$, and 97 in the sodium group $\geq 152.0 \mathrm{mmol} / \mathrm{L}$. Age, baseline Scr, sepsis, surgery, mean arterial pressure, need for mechanical ventilation, Scr, peak Scr, BUN, peak BUN, BG, Mg, prealbumin, albumin, hemoglobin, and AKI stage differed significantly among the 8 groups at the time of AKI diagnosis. Significant interactions were also observed between serum sodium levels and both 90 -day mortality $(P<0.001)$ and 1 -year mortality $(P<0.001)$. The 90 -day mortality rates in the eight sodium interval groups from the lowest $(<130.0$ $\mathrm{mmol} / \mathrm{L})$ to the highest $(\geq 152.0 \mathrm{mmol} / \mathrm{L})$ were $36.1,27.8$, $19.6,24.4,30.7,48.6,52.8$, and $57.7 \%$, respectively. The 1 -year mortality rates in the eight strata were $58.3,47.8$, $33.7,38.9,45.5,64.3,69.4$, and $78.4 \%$, respectively.

\section{Survival Analysis}

Of the patients, 260 (34.9\%) died during the 90-day follow-up. Survival curves for 90-day all-cause mortality across categories of serum sodium are presented in Figure 1. As shown in Table 2, among all the deaths

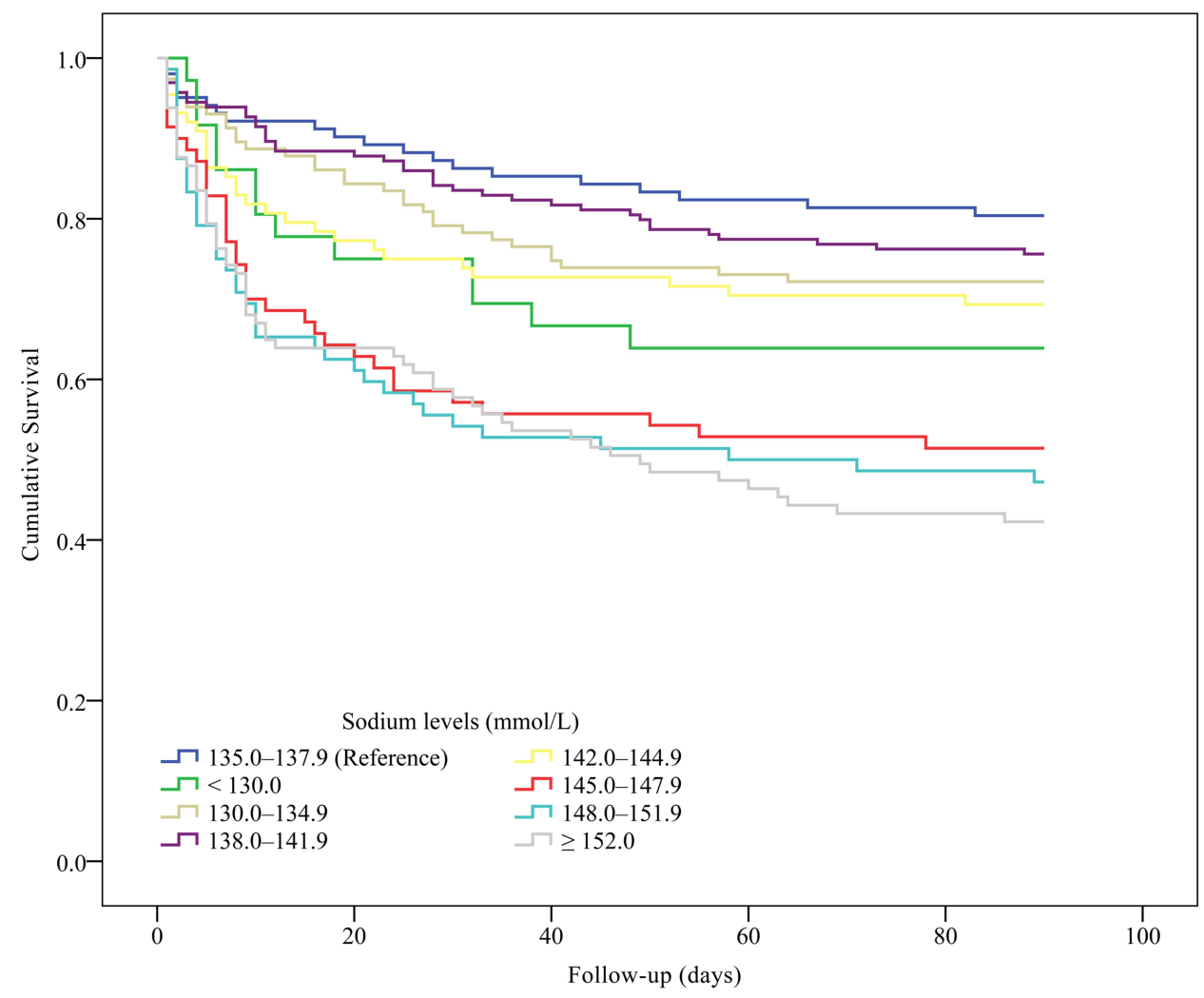

Figure I Kaplan-Meier plot of cumulative rates of 90 -day mortality stratified by serum sodium levels (Log rank test: $P<0.00 \mathrm{I})$. 
Table 2 Association of Categories of Serum Sodium Levels with 90-Day and I-Year Mortality

\begin{tabular}{|c|c|c|c|c|c|c|}
\hline \multirow[t]{2}{*}{ Characteristics } & \multicolumn{3}{|c|}{ 90-Day Outcomes } & \multicolumn{3}{|c|}{ I-Year Outcomes } \\
\hline & $\begin{array}{l}\text { Nonsurvivors } \\
n=260 \text { (34.9) }\end{array}$ & $\begin{array}{c}\text { Survivors } \\
n=484(65.1)\end{array}$ & $\mathbf{P}$ & $\begin{array}{c}\text { Nonsurvivors } \\
n=383(51.8)\end{array}$ & $\begin{array}{c}\text { Survivors } \\
n=356(48.2)\end{array}$ & $P$ \\
\hline Sodium levels (mmol/L) & & & $<0.001$ & & & $<0.001$ \\
\hline$<130.0$ & $13(5.0)$ & $23(4.8)$ & & $21(5.5)$ & $15(4.2)$ & \\
\hline $130.0-134.9$ & $32(12.3)$ & $83(17.1)$ & & $54(14.1)$ & $59(16.6)$ & \\
\hline 135.0-137.9 & $20(7.7)$ & $82(16.9)$ & & $34(8.9)$ & $67(18.8)$ & \\
\hline $138.0-141.9$ & $40(15.4)$ & $124(25.6)$ & & $63(16.4)$ & $99(27.8)$ & \\
\hline $142.0-144.9$ & $27(10.4)$ & $61(12.6)$ & & $40(10.4)$ & $48(13.5)$ & \\
\hline $145.0-147.9$ & $34(13.1)$ & $36(7.4)$ & & 45 (II.7) & $25(7.0)$ & \\
\hline$|48.0-15| .9$ & $38(14.6)$ & $34(7.0)$ & & $50(13.1)$ & $22(6.2)$ & \\
\hline$\geq 152.0$ & $56(2 \mid .5)$ & $4 \mid(8.5)$ & & 76 (19.8) & 21 (5.9) & \\
\hline
\end{tabular}

Note: Values are $\mathrm{n}(\%)$.

from AKI, $5.0 \%$ of the patients had sodium levels $<130.0 \mathrm{mmol} / \mathrm{L}, 12.3 \%$ had sodium levels from 130.0 to $134.9 \mathrm{mmol} / \mathrm{L}, 7.7 \%$ had sodium levels from 135.0 to $137.9 \mathrm{mmol} / \mathrm{L}, 15.4 \%$ had sodium levels from 138.0 to $141.9 \mathrm{mmol} / \mathrm{L}, 10.4 \%$ had sodium levels from 142.0 to $144.9 \mathrm{mmol} / \mathrm{L}, 13.1 \%$ had sodium levels from 145.0 to $147.9 \mathrm{mmol} / \mathrm{L}, 14.6 \%$ had sodium levels from 148.0 to $151.9 \mathrm{mmol} / \mathrm{L}$, and $21.5 \%$ had sodium levels $\geq 152.0 \mathrm{mmol} / \mathrm{L}$.
During the 1-year follow-up, 5 patients were lost, and 383 (51.8\%) died. Survival curves for 1-year all-cause mortality across categories of serum sodium are presented in Figure 2. Among all the deaths from AKI, 5.5\% of the patients had sodium levels $<130.0 \mathrm{mmol} / \mathrm{L}, 14.1 \%$ had sodium levels from 130.0 to $134.9 \mathrm{mmol} / \mathrm{L}, 8.9 \%$ had sodium levels from 135.0 to $137.9 \mathrm{mmol} / \mathrm{L}, 16.4 \%$ had sodium levels from 138.0 to 141.9 $\mathrm{mmol} / \mathrm{L}, 10.4 \%$ had sodium levels from 142.0 to $144.9 \mathrm{mmol} /$ L, $11.7 \%$ had sodium levels from 145.0 to $147.9 \mathrm{mmol} / \mathrm{L}$,

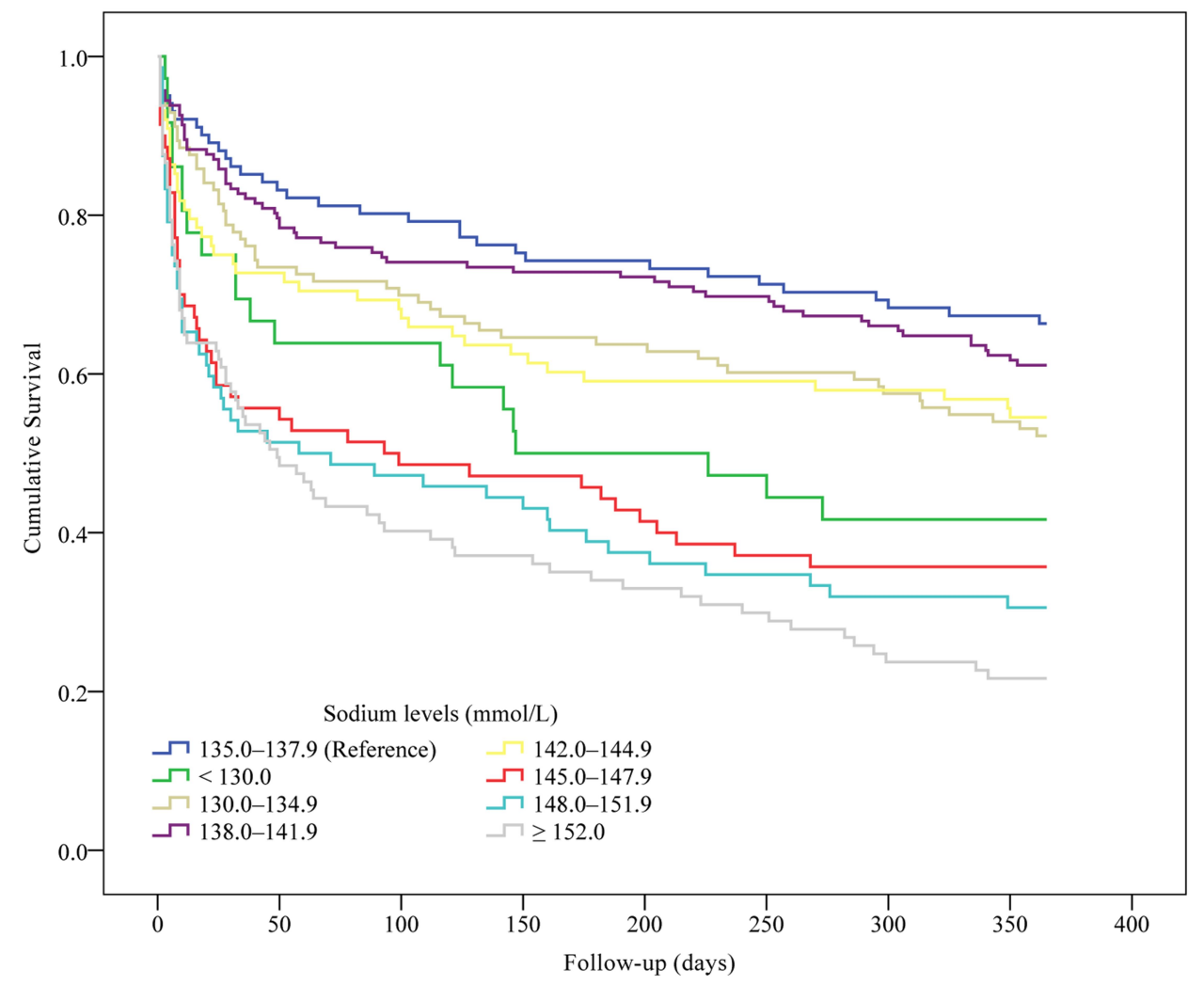

Figure 2 Kaplan-Meier plot of cumulative rates of I-year mortality stratified by serum sodium levels (Log rank test: $P<0.00 \mathrm{I})$. 
$13.1 \%$ had sodium levels from 148.0 to $151.9 \mathrm{mmol} / \mathrm{L}$, and $19.8 \%$ had sodium levels $\geq 152.0 \mathrm{mmol} / \mathrm{L}$.

In the multivariable adjusted analysis, patients with sodium levels $<130.0 \mathrm{mmol} / \mathrm{L}$ [hazard ratio (HR): 2.247 ; 95\% confidence interval (CI): $1.117-4.521 ; P=0.023]$, from 142.0 to $144.9 \mathrm{mmol} / \mathrm{L}$ (HR: $1.964 ; 95 \%$ CI: $1.100-3.508$; $P=0.022$ ), from 145.0 to $147.9 \mathrm{mmol} / \mathrm{L}$ (HR: $2.942 ; 95 \% \mathrm{CI}$ : 1.693-5.114; $P<0.001$ ), from 148.0 to $151.9 \mathrm{mmol} / \mathrm{L}$ (HR: 3.455; 95\% CI: $2.009-5.944 ; P<0.001)$, and $\geq 152.0 \mathrm{mmol} / \mathrm{L}$ (HR: 3.587; 95\% CI: 2.151-5.983; $P<0.001$ ) had relatively higher 90-day mortality rates (Table 3 ).

Significant interactions were also observed between serum sodium levels and 1-year mortality: patients with sodium levels $<130.0 \mathrm{mmol} / \mathrm{L}$ (HR: $1.944 ; 95 \% \mathrm{CI}$ : 1.$125-3.360 ; P=0.017$ ), from 142.0 to $144.9 \mathrm{mmol} / \mathrm{L}$ (HR: $1.681 ; 95 \%$ CI: $1.062-2.660 ; P=0.027)$, from 145.0 to $147.9 \mathrm{mmol} / \mathrm{L} \quad$ (HR: 2.631; 95\% CI: $1.683-4.112$; $P<0.001$ ), from 148.0 to $151.9 \mathrm{mmol} / \mathrm{L}$ (HR: $2.411 ; 95 \%$ CI: $1.552-3.744 ; P<0.001$ ), and $\geq 152.0 \mathrm{mmol} / \mathrm{L}$ (HR: 3.037; 95\% CI: $2.021-4.563 ; P<0.001)$ had relatively higher 1-year mortality rates (Table 3 ).

\section{Discussion}

Sodium is the main electrolyte responsible for serum osmolarity. ${ }^{17}$ Patients suffering from AKI have impaired sodium homeostasis. Dysnatremia is the most common electrolyte abnormality in clinical practice, as our study found $(52.4 \%) .{ }^{11}$ No studies have independently or simultaneously investigated the prognostic role of such electrolytes among elderly patients with AKI. This study analyzed the 90-day mortality and 1-year mortality risks in relation to different serum sodium intervals in elderly AKI patients. It was not surprising that sodium levels outside the normal range $(\mathrm{Na}<130.0$ and $>145.0 \mathrm{mmol} / \mathrm{L})$ were associated with an increased mortality risk; it was unexpected that one normal sodium level (Na: 142.0-144.9 mmol/L) was also associated with a significantly increased mortality risk in AKI patients.

In the elderly population, the incidence of electrolyte imbalance is expected to be higher because of organ decline, decreased physiologic reserves, frailty, and the higher prevalence of disability and functional impairment. ${ }^{18-20}$ Kidneys are responsible for maintaining water homeostasis, and AKI could magnify the effect of dysnatremia on the clinical consequences. Normal ranges of serum sodium levels within $135.0-145.0 \mathrm{mmol} / \mathrm{L}$ used in clinical settings are determined mainly based on healthy subjects, and whether these values are applicable to elderly patients with complex comorbidities is still unknown. ${ }^{21}$ Through interfering with tubular functions, AKI disturbs the physiological regulation of electrolyte homeostasis, which could be more prominent in the elderly population. Previous studies have consistently reported that electrolyte imbalances, including dysnatremia and dyskalemia, are associated with worse in-hospital outcomes. Furthermore, the adverse impact of dysnatremia on clinical outcomes might be independent of the presence of initial AKI.

Hypernatremia is characterized by a deficit of total body water relative to the total body sodium level and is defined as a serum sodium concentration exceeding $145 \mathrm{mmol} / \mathrm{L}{ }^{22}$ Hypernatremia in the geriatric population is a common disorder associated with significant mortality. Older people are

Table 3 Multivariate Cox Proportional Hazard Model Analysis of Risk Factors for Mortality

\begin{tabular}{|c|c|c|c|c|c|c|}
\hline \multirow[t]{2}{*}{ Risk Factors } & \multicolumn{3}{|c|}{ 90-Day Mortality } & \multicolumn{3}{|c|}{ I-Year Mortality } \\
\hline & HR & $95 \% \mathrm{Cl}$ & $\boldsymbol{P}$ & HR & $95 \% \mathrm{Cl}$ & $P$ \\
\hline MAP & 0.960 & $0.95 \mathrm{I}-0.968$ & $<0.001$ & 0.979 & $0.972-0.986$ & $<0.001$ \\
\hline Albumin & - & - & - & 0.906 & $0.887-0.924$ & $<0.001$ \\
\hline Sodium levels (mmol/L) & & & $<0.001$ & & & $<0.001$ \\
\hline $135.0-137.9$ & Reference & Reference & - & Reference & Reference & - \\
\hline$<130.0$ & 2.247 & $1.117-4.521$ & 0.023 & 1.944 & $1.125-3.360$ & 0.017 \\
\hline $130.0-134.9$ & 1.442 & $0.825-2.522$ & 0.199 & 1.436 & $0.935-2.207$ & 0.099 \\
\hline $138.0-14 \mid .9$ & 1.438 & $0.840-2.463$ & 0.186 & 1.428 & $0.938-2.173$ & 0.097 \\
\hline $142.0-144.9$ & 1.964 & $1.100-3.508$ & 0.022 & 1.681 & $1.062-2.660$ & 0.027 \\
\hline $145.0-147.9$ & 2.942 & $1.693-5.114$ & $<0.001$ & 2.631 & $1.683-4.112$ & $<0.001$ \\
\hline $148.0-151.9$ & 3.455 & $2.009-5.944$ & $<0.001$ & $2.41 I$ & $1.552-3.744$ & $<0.001$ \\
\hline$\geq 152.0$ & 3.587 & $2.15 \mathrm{I}-5.983$ & $<0.001$ & 3.037 & $2.021-4.563$ & $<0.001$ \\
\hline
\end{tabular}

Abbreviations: MAP, mean arterial pressure; $\mathrm{Cl}$, confidence interval; $\mathrm{HR}$, hazard ratio. 
predisposed to developing hypernatremia because of agerelated physiologic changes such as decreased thirst drive, impaired urinary concentrating ability, and reduced total body water. ${ }^{23}$ Medications may also exacerbate this predisposition. Clinical data reported that hypernatremia is present in approximately $2.0 \%$ of patients older than 65 years but in almost $4.8 \%$ of those older than 75 years admitted to the hospital. ${ }^{24}$ In a retrospective study involving 8,441 older patients admitted to the ICU, approximately $3.6 \%$ had hypernatremia on admission, and $15.3 \%$ developed hypernatremia during hospitalization; ${ }^{25}$ the mortality rate in those admitted with hypernatremia was approximately $33.3 \%$, while patients without hypernatremia had a mortality rate of $18.1 \% .^{25}$ If patients had normal serum sodium levels on admission and developed mild hypernatremia (145.0-150.0 $\mathrm{mmol} / \mathrm{L})$ or moderate to severe hypernatremia $(>150.0 \mathrm{mmol} / \mathrm{L})$ in the ICU, the mortality rates increased to $29.5 \%$ and $46.2 \%$, respectively. ${ }^{25}$ Other studies have confirmed these findings: mortality increases with increasing serum sodium level, and even mild hypernatremia is associated with significant mortality in hospitalized elderly patients, even after accounting for confounders. $^{25-27}$

Hyponatremia, defined as a serum sodium concentration below $135 \mathrm{mmol} / \mathrm{L}$, is the most common electrolyte problem seen in hospitalized patients. ${ }^{22}$ Advanced age is an independent risk factor for the development of hyponatremia due to the decreased ability of the body to handle stresses related to salt and water balance. Moreover, drugs such as thiazides and nonsteroidal anti-inflammatory agents commonly used in this age group contribute to a decreased renal ability to excrete free water. Critically ill patients also demonstrate impaired renal capacity to excrete free water. Hyponatremia has been observed in $14.5-37.9 \%$ of patients upon hospital admission, ${ }^{22,28,29}$ while rates of hospital-acquired hyponatremia range from 11.0 to $38.2 \% .^{28,30,31}$ Mortality rates vary significantly based on the population studied, and in several studies, hyponatremia has been associated with an increased risk of death. ${ }^{22,28-31}$

Critically ill patients often receive large volumes of fluids. Diuretics in such patients minimize fluid overload and might increase the urine output. However, diuretics may decrease the effective circulating volume and may cause a decrease in renal blood flow and GFR or even aggravation of kidney dysfunction. Hence, the treatment and prevention of AKI remains a major challenge for intensive care physicians and nephrologists. While caring for AKI patients, the focus should be on identifying any reversible causes of renal damage, stopping any potential nephrotoxic agents, optimizing hemodynamics, and correcting electrolyte abnormalities. When managing severe AKI, whether to provide renal replacement therapy (RRT) and, if it is needed, when to initiate it are unclear. It is widely accepted that if there are life-threatening complications of AKI, such as hyperkalemia or metabolic acidosis, RRT should be initiated immediately. However, in the absence of such complications, the appropriate timing of the initiation of RRT remains unclear. On the basis of the current evidence, therefore, the use of diuretics should be limited to the management of volume overload and/or hyperkalemia. Patients should undergo RRT promptly when indicated and the use of diuretics should not delay the institution of RRT. A significant interaction between serum sodium levels and mortality was observed in our study ( $P$ interaction: $<0.001$ for 90 -day mortality and $<0.001$ for 1-year mortality). Across different sodium strata, higher and lower serum sodium levels were both associated with an increased risk of mortality among patients with AKI. Hypernatremia has been poorly assessed in AKI, probably because according to a previous report, more AKI patients suffer from hyponatremia $\left(\mathrm{Na}^{+}<135 \mathrm{mmol} / \mathrm{L}\right)$ than hypernatremia $\left(\mathrm{Na}^{+}>\right.$ $145 \mathrm{mmol} / \mathrm{L})(27.0 \%$ vs $5.7 \%)$ when admitted to the ICU. ${ }^{32}$ However, the percentage of patients with hypernatremia was $32.1 \%$ compared with $20.3 \%$ of the patients with hyponatremia in our study. Both hyponatremia and hypernatremia can have direct adverse effects on the function of various organs, including the brain, heart, and musculoskeletal system, and subsequently increase mortality. Dysnatremia may be a potential target for correction for clinicians. However, whether correcting dysnatremia could improve outcomes should be evaluated in future studies.

\section{Strengths and Limitations}

There were also several limitations of this study. First, this was a single-center retrospective analysis, and we could only establish an association rather than a causal relationship. In addition, different results could be reached when using patient records from other centers. Therefore, subject selection bias cannot be ignored. Second, we only analyzed sodium levels at the time of AKI diagnosis, and these levels could change over time (eg, initiating electrolyte therapy). Third, although we attempted to account for a number of potential confounders in the multivariable analyses, it is plausible that other unidentified variables 
may have influenced the results. Finally, we used all-cause mortality rather than cause-specific mortality, which was also a major limitation.

\section{Conclusion}

Sodium levels outside the interval of 130.0-141.9 mmol/L were associated with increased 90-day mortality and 1 -year mortality risks in elderly AKI patients.

\section{Abbreviations}

AKI, acute kidney injury; KDIGO, Kidney Disease Improving Global Outcomes; Scr, serum creatinine; eGFR, estimated glomerular filtration rate; MAP, mean arterial pressure; $\mathrm{MV}$, mechanical ventilation; $\mathrm{BUN}$, blood urea nitrogen; CRP, C-reactive protein; RRT, renal replacement therapy.

\section{Data Sharing Statement}

The datasets used and/or analyzed during the current study are available from the corresponding author on reasonable request.

\section{Ethical Approval}

This study has been approved by the Ethics Committee of the Chinese PLA General Hospital (number: S2017-054-01).

\section{Informed Consent}

The requirement to obtain written informed consent from each patient was waived because this was an observational retrospective study.

\section{Acknowledgments}

This manuscript was edited for English language by American Journal Experts (AJE).

\section{Funding}

This study was funded by grants from the National Natural Science Foundation of China (grant 81871587 to Dr FHZ), the Special Scientific Research Project of Military Health Care (grant 20BJZ27 to Dr FHZ), and the Military Medical Innovation Project (grant 18CXZ026 and CX10010 to Dr FHZ).

\section{Disclosure}

The authors declare that they have no competing interests.

\section{References}

1. Lee SA, Cozzi M, Bush EL, et al. Distant organ dysfunction in acute kidney injury: a review. Am J Kidney Dis. 2018;72(6):846-856. doi:10.1053/j.ajkd.2018.03.028

2. Yang L, Xing G, Wang L, et al. Acute kidney injury in China: a cross-sectional survey. Lancet. 2015;386(10002):1465-1471. doi:10.1016/S0140-6736(15)00344-X

3. Anderson S, Eldadah B, Halter JB, et al. Acute kidney injury in older adults. J Am Soc Nephrol. 2011;22(1):28-38. doi:10.1681/ASN.201 0090934

4. Hoste EAJ, Kellum JA, Selby NM, et al. Global epidemiology and outcomes of acute kidney injury. Nat Rev Nephrol. 2018;14 (10):607-625.

5. Moore PK, Hsu RK, Liu KD. Management of acute kidney injury: core curriculum 2018. Am J Kidney Dis. 2018;72(1):136-148. doi:10.1053/j.ajkd.2017.11.021

6. Rewa O, Bagshaw SM. Acute kidney injury-epidemiology, outcomes and economics. Nat Rev Nephrol. 2014;10(4):193-207.

7. Denic A, Glassock RJ, Rule AD. Structural and functional changes with the aging kidney. Adv Chronic Kidney Dis. 2016;23(1):19-28.

8. Chao C-T, Tsai H-B, Lin Y-F, et al. Acute kidney injury in the elderly: only the tip of the iceberg. $J$ Clin Gerontol Geriatric. 2014;5(1):7-12.

9. Kellum JA, Chawla LS. Acute kidney injury: still deadly 10 years later. Am J Respir Crit Care Med. 2014;189(9):1016-1017.

10. Doyle JF, Forni LG. Acute kidney injury: short-term and long-term effects. Crit Care. 2016;20(1):188. doi:10.1186/s13054-016-1353-y

11. Sun L, Hou Y, Xiao Q, et al. Association of serum sodium and risk of all-cause mortality in patients with chronic kidney disease: a meta-analysis and sysematic review. Sci Rep. 2017;7(1):15949. doi:10.1038/s41598-017-16242-3

12. Gao XP, Zheng CF, Liao MQ, et al. Admission serum sodium and potassium levels predict survival among critically ill patients with acute kidney injury: a cohort study. BMC Nephrol. 2019;20(1):311. doi:10.1186/s12882-019-1505-9

13. Kidney Disease: Improving Global Outcomes (KDIGO) Acute Kidney Injury Work Group. KDIGO clinical practice guideline for acute kidney injury. Kidney Int Suppl. 2012;2:1-138.

14. Levey AS, Stevens LA, Schmid CH, et al. A new equation to estimate glomerular filtration rate. Ann Intern Med. 2009;150 (9):604-612. doi:10.7326/0003-4819-150-9-200905050-00006

15. Chao CT, Tsai HB, Wu CY, et al. The severity of initial acute kidney injury at admission of geriatric patients significantly correlates with subsequent in-hospital complications. Sci Rep. 2015;5(1):13925. doi:10.1038/srep13925

16. Levy MM, Evans LE, Rhodes A. The surviving sepsis campaign bundle: 2018 update. Intensive Care Med. 2018;44(6):925-928. doi:10.1007/s00134-018-5085-0

17. Mc Causland FR, Wright J, Waikar SS. Association of serum sodium with morbidity and mortality in hospitalized patients undergoing major orthopedic surgery. J Hosp Med. 2014;9(5):297-302. doi: $10.1002 / \mathrm{jhm} .2168$

18. Abdel-Kader K, Girard TD, Brummel NE, et al. Acute kidney injury and subsequent frailty status in survivors of critical illness: a secondary analysis. Crit Care Med. 2018;46(5):e380-e388. doi:10.1097/CCM.0000000000003003

19. Baek SH, Lee SW, Kim SW, et al. Frailty as a predictor of acute kidney injury in hospitalized elderly patients: a single center, retrospective cohort study. PLoS One. 2016;11(6):e0156444. doi:10.1371/ journal.pone. 0156444

20. Muscedere J, Waters B, Varambally A, et al. The impact of frailty on intensive care unit outcomes: a systematic review and meta-analysis. Intensive Care Med. 2017;43(8):1105-1122. doi:10.1007/s00134017-4867-0 
21. Polcwiartek C, Hansen SM, Kragholm K, et al. Prognostic role of serum sodium levels across different serum potassium levels in heart failure patients: a Danish register-based cohort study. Int J Cardiol. 2018;272:244-249. doi:10.1016/j.ijcard.2018.08.045

22. Funk GC, Lindner G, Druml W, et al. Incidence and prognosis of dysnatremias present on ICU admission. Intensive Care Med. 2010;36(2):304-311. doi:10.1007/s00134-009-1692-0

23. Taffet G, Shah M, Workeneh B. Hypernatremia in the geriatric population. Clin Interv Aging. 2014;1987. doi:10.2147/CIA. S65214

24. Turgutalp K, Ozhan O, Gok Oguz E, et al. Community-acquired hypernatremia in elderly and very elderly patients admitted to the hospital: clinical characteristics and outcomes. Med Sci Monit. 2012;18(12):CR729-734. doi:10.12659/MSM.883600

25. Darmon M, Timsit J-F, Francais A, et al. Association between hypernatraemia acquired in the ICU and mortality: a cohort study. Nephrol Dial Transplant. 2010;25(8):2510-2515. doi:10.1093/ndt/gfq067

26. Hoorn EJ, Betjes MG, Weigel J, et al. Hypernatraemia in critically ill patients: too little water and too much salt. Nephrol Dial Transplant. 2008;23(5):1562-1568. doi:10.1093/ndt/gfm831
27. Lindner G, Funk GC, Schwarz C, et al. Hypernatremia in the critically ill is an independent risk factor for mortality. Am J Kidney Dis. 2007;50(6):952-957. doi:10.1053/j.ajkd.2007.08.016

28. Wald R, Jaber BL, Price LL, et al. Impact of hospital-associated hyponatremia on selected outcomes. Arch Intern Med. 2010;170 (3):294-302. doi:10.1001/archinternmed.2009.513

29. Waikar SS, Mount DB, Curhan GC. Mortality after hospitalization with mild, moderate, and severe hyponatremia. Am J Med. 2009;122 (9):857-865. doi:10.1016/j.amjmed.2009.01.027

30. Stelfox HT, Ahmed SB, Zygun D, et al. Characterization of intensive care unit acquired hyponatremia and hypernatremia following cardiac surgery. Can J Anaesth. 2010;57(7):650-658. doi:10.1007/s12630010-9309-1

31. Stelfox HT, Ahmed SB, Khandwala F, et al. The epidemiology of intensive care unit-acquired hyponatraemia and hypernatraemia in medical-surgical intensive care units. Crit Care. 2008;12(6):R162. doi:10.1186/cc7162

32. Peres LA, Wandeur V, Matsuo T. Predictors of acute kidney injury and mortality in an intensive care unit. J Bras Nefrol. 2015;37 (1):38-46. doi:10.5935/0101-2800.20150007

\section{Publish your work in this journal}

The International Journal of General Medicine is an international, peer-reviewed open-access journal that focuses on general and internal medicine, pathogenesis, epidemiology, diagnosis, monitoring and treatment protocols. The journal is characterized by the rapid reporting of reviews, original research and clinical studies across all disease areas. The manuscript management system is completely online and includes a very quick and fair peer-review system, which is all easy to use. Visit http://www.dovepress.com/ testimonials.php to read real quotes from published authors. 